3 Kulka K, Hatfull G, Ojha AK. Growth of Mycobacterium tuberculosis biofilms. J Vis Exp 2012; 60: 3820.

4 Falkinham III JO, Falkinham JO. Surrounded by mycobacteria: nontuberculous mycobacteria in the human environment. J Appl Microbiol 2009; 107: 356-367.

5 Falkinham JO. Ecology of nontuberculous mycobacteria - where do human infections come from? Semin Respir Crit Care Med 2013; 34: 95-102.

6 Leung JM, Olivier KN. Nontuberculous mycobacteria: the changing epidemiology and treatment challenges in cystic fibrosis. Curr Opin Pulm Med 2013; 19: 662-669.

7 Qvist T, Gilljam M, Jönsson B, et al. Epidemiology of nontuberculous mycobacteria among patients with cystic fibrosis in Scandinavia. J Cyst Fibros 2015; 14: 46-52.

8 Qvist T, Pressler T, Thomsen VO, et al. Nontuberculous mycobacterial disease is not a contraindication to lung transplantation in patients with cystic fibrosis: a retrospective analysis in a Danish patient population. Transpl Proc 2013; 45: 342-345.

9 Marsollier L, Brodin P, Jackson M, et al. Impact of Mycobacterium ulcerans biofilm on transmissibility to ecological niches and Buruli ulcer pathogenesis. PLoS Pathog 2007; 3: e62.

10 Fazli M, Bjarnsholt T, Kirketerp-Møller K, et al. Nonrandom distribution of Pseudomonas aeruginosa and Staphylococcus aureus in chronic wounds. J Clin Microbiol 2009; 47: 4084-4089.

11 Høiby N, Bjarnsholt T, Moser C, et al. ESCMID guideline for the diagnosis and treatment of biofilm infections 2014. Clin Microbiol Infect 2015; 21: Suppl. 1, S1-S25.

12 Ciofu O, Nielsen TT, Jensen PØ, et al. Antimicrobial resistance, respiratory tract infections and role of biofilms in lung infections in cystic fibrosis patients. Adv Drug Deliv Rev 2015; 85: 7-23.

13 Bjarnsholt T, Jensen PO, Fiandaca MJ, et al. Pseudomonas aeruginosa biofilms in the respiratory tract of cystic fibrosis patients. Pediatr Pulmonol 2009; 44: 547-558.

14 Kolpen M, Kragh KN, Bjarnsholt T, et al. Denitrification by cystic fibrosis pathogens - Stenotrophomonas maltophilia is dormant in sputum. Int J Med Microbiol 2015; 305: 1-10.

15 Howard ST, Rhoades E, Recht J, et al. Spontaneous reversion of Mycobacterium abscessus from a smooth to a rough morphotype is associated with reduced expression of glycopeptidolipid and reacquisition of an invasive phenotype. Microbiology 2006; 152: 1581-1590.

\title{
Cost-effectiveness of incorporating bedaquiline into a treatment regimen for MDR/XDR-TB in Germany
}

The emergence of multi-drug-resistant (MDR) and extensively drug-resistant (XDR) tuberculosis (TB) has re-established TB as a major worldwide health concern [1-3]. Compared with conventional TB drug regimens, treatments for MDR-TB and XDR-TB are more toxic, expensive and associated with poorer outcomes [3, 4]. In Germany, 102 (2.4\%) of the 4318 TB cases reported for 2013 were MDR-TB, including 10 patients with XDR-TB [5], increasing from $54(1.8 \%)$ cases in 2011 [5, 6]. An important factor behind the increase in Germany is migration, with more cases occurring in patients from the former Soviet Union than in native Germans [5].

Bedaquiline (BDQ) is a novel treatment that may be added for 24 weeks to a World Health Organization (WHO) recommended antibiotic background regimen (BR) for the treatment of MDR-TB [7]. Wolfson et al. [8] developed a comprehensive model looking at cost-effectiveness of BDQ plus BR (BDQ+BR) in the UK, over a substantially longer time horizon than a previously published WHO analysis $[8,9]$.

In this study, we report findings from an adapted version examining cost-effectiveness in the German healthcare system, where typically routine hospitalisation of patients with MDR-TB is carried out. A previous study of patients with MDR- TB in Germany found the total cost of treating to be $€ 64429.23$ [10].

After adjustment to the perspective of the German Statutory Health Insurance (SHI) system, a cohort-based Markov model was used to estimate the cost-effectiveness of BDQ as an add-on for 24 weeks to a weighted antibiotic BR containing pyrazinamide, one injectable, one fluoroquinolone and one WHO group 4 agent, taking into account different strain resistance patterns as described in the C208 trial and following local guidelines $[4,7,11]$. This regimen of $\mathrm{BDQ}+\mathrm{BR}$ was then compared with $\mathrm{BR}$ alone for the treatment of MDR- and XDR-TB. Comparison of BDQ with Delamanid, an alternative treatment option that was introduced later [12], was outside the focus of our analysis. Costs and effectiveness were discounted at an annual rate of 3\% [13]. The time horizon for the model was 10 years. The model state structure was designed to reflect initial clinical care in patients with TB. 
The goal of drug treatment in the simulation was to induce the sputum culture converted state (SCC) in the sputum culture positive patient cohort and maintain the SCC until treatment completion (and thus assumed to be cured of MDR-TB) [14].

Patients failing to achieve SCC during the first year were considered treatment failures and were transited to the "active secondary MDR-TB" state by no later than month 12 of the long-term MDR-treatment, to begin a new treatment. Patients who failed to achieve SCC with the new treatment were assumed to occupy the same state until death or loss to follow-up for simplifying the model structure. Still, it is valid to consider further treatment options with a third-line or even fourth-line treatment, but these were outside the scope of our model when examining MDR-TB. The probability of death was assumed to be positively dependent on the sputum status of the cohort (active TB versus SCC versus cured), their status in terms of MDR- and XDR-TB, and whether they were lost to follow-up.

A cohort of 65 MDR-TB patients was included in the model simulation, reflecting current epidemiological data for Germany at the time of model design. Of these, $87.7 \%$ were assumed to have been treated in hospital for a mean duration of 89.1 days [10].

Inputs into the model for clinical outcomes (transition probabilities) and dosages were derived from a randomised, placebo-controlled phase IIb trial of BDQ [15], and an open-label, single-arm phase IIb study of BDQ efficacy [16]. Other model parameters, including the costs of the regimen under consideration, were adapted either from a previous German study [10] or international literature where data specific to Germany were not available. According to the approval criteria of BDQ, any replacement of other drugs from the BR with BDQ was not taken into consideration.

Inpatient costs were based on data from the German G-DRG system, with reimbursement dependent upon disease type and severity, as well as the cumulative time spent in hospital [17]. Health utility weights were adapted from a previous study in a low incidence setting, as local data for Germany are not available [9]. Deterministic sensitivity analyses were conducted to assess the impact of varying model assumptions and parameters by $\pm 20 \%$ on model results, in terms of utility, clinical parameters, transition probabilities and drug costs, following international recommendations [18].

Using a combination therapy $(\mathrm{BDQ}+\mathrm{BR})$ for a cohort of 65 patients with MDR-TB over a 10-year time period, the results demonstrated substantially better quality-adjusted life-year (QALY) outcomes compared with treatment with BR alone (incremental outcome of 73.29 QALYs gained with an incremental cost-effectiveness ratio of $€ 22118$ for a 3\% discount rate). Furthermore, this result was seen despite increased total costs for the cohort associated with BDQ+BR compared with BR alone (€5583515 and €3962499, respectively). In addition to QALY gains, treatment with BDQ+BR led to an extra incremental 74.58 life-years gained compared with those treated with BR alone (incremental cost of $€ 21736$ per life-year gained (LYG)).

A cost-effectiveness analysis was performed for a cohort of 10 patients with XDR-TB according to current epidemiological data at the time of model design [5] and based on efficacy data from the open-label phase II study C209 [19]. In this trial, BDQ was given as an add-on for 24 weeks to a weighted, individualised $\mathrm{BR}$ containing at least one injectable, one fluoroquinolone and one WHO group 4 agent, taking into account individual strain resistance patterns. Findings from this trial indicated that adding BDQ as described also yields comparable rates of SCC in patients with XDR-TB, which was confirmed in real world settings $[20,21]$. Although $B D Q+B R$ was associated with higher total costs compared with BR alone ( $€ 1662746$ versus $€ 1609831$, respectively), treatment led to better outcomes both in total QALYs (29.72 versus 20.57$)$ and LYG (40.72 versus 31.05$)$. The incremental cost per LYG were $€ 5780.54$ and $€ 5472.13$, respectively, suggesting that $\mathrm{BDQ}+\mathrm{BR}$ is more cost-effective than $\mathrm{BR}$ alone, and more cost-effective compared with treatment of a MDR-TB cohort.

Multiple deterministic sensitivity analyses were performed to ensure the model robustness and to assess the impact of individual parameters. The data suggest that the hazard ratio of SCC is the most influential parameter in the model, with changes to the input of $\pm 20 \%$ resulting in changes to the cost per QALY of $39 \%$ and $-20 \%$, respectively. Changes to all other input parameters had a very minor impact on the results $(<3 \%$ change to the cost per QALY).

The probability that $\mathrm{BDQ}+\mathrm{BR}$ is cost-effective versus $\mathrm{BR}$ alone at an affordability threshold of $€ 30000$ per QALY gained was 67\% (fig. 1). When adapting the British thresholds used for orphan drugs of $£ 50000$ per QALY gained ( $€ 63400,2014$ conversion values), the probability of BDQ being cost-effective versus BR was $95 \%$.

A previous study described a model that demonstrated the cost-effectiveness of adding BDQ to a BR from the UK healthcare perspective [9]. The present German adaptation of this model demonstrates that adding BDQ to a BR for the treatment of MDR-TB proves cost-effective in most scenarios. This finding further reinforces our previously drawn conclusions that the model and its results are generalisable to other high income settings $[9,22]$. 


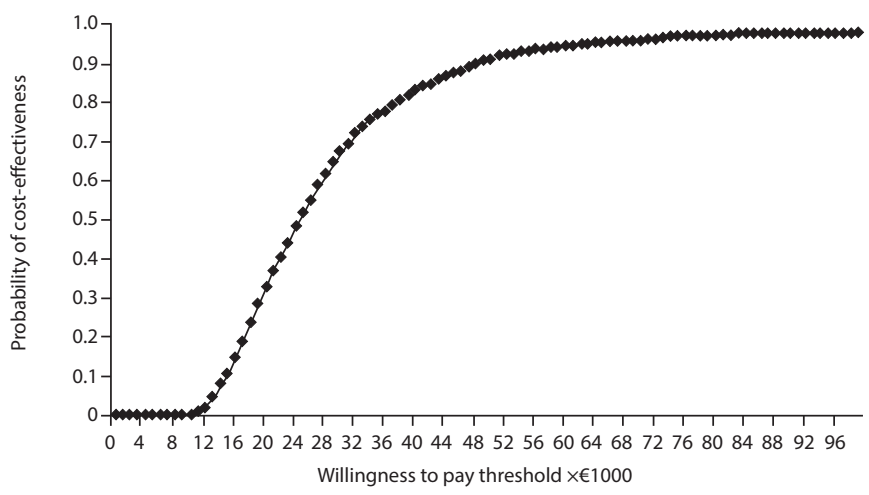

FIGURE 1 Cost-effectiveness acceptability curve for the bedaquiline+background regimen (BR) versus BR alone from a German payer perspective (multidrug- resistant tuberculosis cohort).

As with most other European countries, the prevalence of TB has reached a low but relatively stable level in Germany [5, 6]. However, the resources needed to treat MDR- and XDR-TB imply that these diseases remain a substantial challenge. Our analysis demonstrates that treatment costs for MDR- and XDR-TB are primarily driven by hospitalisation and drug costs. Despite higher drug-related costs (acquisition and monitoring), it is likely that the addition of BDQ will lead to cost savings, primarily due to lower hospitalisation rates and more patients being managed in an outpatient setting in Germany. Such effects may be gained by both a reduction in the time to SCC and a higher proportion of patients achieving SCC when treated with $\mathrm{BDQ}+\mathrm{BR}$ compared with $\mathrm{BR}$ alone.

The model described here is subject to a number of assumptions and limitations. First, the use of trial efficacy data may not accurately reflect results found in German clinical practice. However, recent publications with data from France and South Africa demonstrate that high rates of SCC (up to 97\%) are also obtained in real-world practice for patients with MDR- and XDR-TB [20, 21], thus confirming clinical trial data in at least one cohort which is comparable to the German setting [20]. In contrast to the mortality imbalance observed in the placebo-controlled phase II clinical trial of bedaquiline [15], our model incorporates mortality data derived from literature relating to both general and German-specific values [23-25]. This discrepancy arises because there were no identified reasons that explained the trial-observed mortality imbalance $[7,26]$.

In conclusion, addition of BDQ to the standard of care for treatment of MDR- and XDR-TB is likely to be highly cost-effective from a German SHI perspective.

@ERSpublications

Adding bedaquiline to the background regimen is likely to be cost-effective for treatment of MDR/XDR-TB in Germany http://ow.ly/SwMdv

Lara J. Wolfson ${ }^{1}$, Judith Gibbert ${ }^{2}$, Daniel Wirth ${ }^{3}$ and Roland Diel ${ }^{4}$

${ }^{1}$ Janssen Pharmaceutica NV, Beerse, Belgium. ${ }^{2}$ University of Cologne, Cologne, Germany. ${ }^{3}$ Janssen-Cilag, Neuss, Germany. ${ }^{4}$ University of Kiel, Kiel, Germany.

Correspondence: Daniel Wirth, Health Economics and Market Access, Janssen-Cilag GmbH, Johnson \& Johnson Platz 1, 41470 Neuss, Germany. E-mail: dwirth2@its.jnj.com

Received: May 222015 | Accepted after revision: Aug 272015 | First published online: Oct 222015

Conflict of interest: Disclosures can be found alongside the online version of this article at erj.ersjournals.com

\section{References}

1 Raviglione MC. Issues facing TB control (7). Multiple drug-resistant tuberculosis. Scott Med J 2000; 45: Suppl., $52-55$.

2 Raviglione MC, Smith IM. XDR tuberculosis - implications for global public health. N Engl J Med 2007; 356: 656-659. World Health Oganization. Global Tuberculosis Report 2014. Geneva, World Health Oganization, 2014.

Schaberg T, Bauer T, Castell S, et al. Empfehlungen zur therapie, chemopravention und chemoprophylaxe der tuberkulose im erwachsenen- und kindesalter. Deutsches Zentralkomitee zur Bekampfung der tuberkulose (DZK), Deutsche Gesellschaft fur Pneumologie und Beatmungsmedizin (DGP) [Recommendations for therapy, chemoprevention and chemoprophylaxis of tuberculosis in adults and children. German Central Committee against Tuberculosis (DZK), German Respiratory Society (DGP)]. Pneumologie 2012; 66: 133-171.

5 Robert-Koch-Institut. Bericht zur Epidemiologie der Tuberkulose in Deutschland für 2012. www.rki.de/DE/Content/ InfAZ/T/Tuberkulose/Download/TB2012.html Date last accessed: March 17, 2015. Date last updated: March 17, 2014. 
6 Robert-Koch-Institut. Bericht zur Epidemiologie der Tuberkulose in Deutschland für 2013. www.rki.de/DE/ Content/InfAZ/T/Tuberkulose/Download/TB2013.pdf?_blob=publicationFile Date last accessed: February 12, 2015. Date last updated: December 12, 2014.

7 World Health Oganization. The Use of Bedaquiline in the Treatment of Multidrug-Resistant Tuberculosis: Interim Policy Guidance. Geneva, World Health Oganization, 2013.

8 Wolfson LJ, Walker A, Hettle R, et al. Cost-effectiveness of adding bedaquiline to drug regimens for the treatment of multidrug-resistant tuberculosis in the UK. PLoS One 2015; 10: e0120763.

9 Vassell A. Cost-Effectiveness of Introducing Bedaquiline in MDR-TB Regimens - A Exploratory Analysis. http://who.int/ tb/challenges/mdr/CEA_bdqreport_final.pdf Date last accessed: February 17, 2015. Date last updated: January 26, 2015.

10 Diel R, Nienhaus A, Lampenius $\mathrm{N}$, et al. Cost of multi drug resistance tuberculosis in Germany. Respir Med 2014; 108: $1677-1687$

11 Diacon AH, Pym A, Grobusch MP, et al. Multidrug-resistant tuberculosis and culture conversion with bedaquiline. $N$ Engl J Med 2014; 371: 723-732.

12 Schaberg T, Otto-Knapp R, Bauer T. Kurze ubersicht uber neue medikamente zur therapie der multiresistenten tuberkulose [New drugs for the treatment of multidrug-resistant tuberculosis (MDR-TB)]. Pneumologie 2015; 69: $282-286$.

13 Institut für Qualität und Wirtschaftlichkeit im Gesundheitswesen. 2009. Arbeitspapier Kostenbestimmung. www.iqwig.de/download/Arbeitspapier_Kostenbestimmung_v_1_0.pdf Date last accessed: March 17, 2015. Date last updated April 22, 2015.

14 World Health Oganization. Multidrug and Extensively Drug-Resistant TB (M/XDR-TB): 2010 Global Report on Surveillance and Response. Geneva, World Health Oganization, 2010.

15 Diacon AH, Pym A, Grobusch MP, et al. Multidrug-resistant tuberculosis and culture conversion with bedaquiline. N Engl J Med 2014; 371: 723-732.

16 Janssen. Anti-Infective Drug Advisory Committee Meeting Briefing Document: TMC207 (bedaquiline) Treatment of Patients with MDR-TB. www.fda.gov/downloads/AdvisoryCommittees/CommitteesMeetingMaterials/Drugs/ Anti-InfectiveDrugsAdvisoryCommittee/UCM329260.pdf Date last accessed: March 17, 2015. Date last updated: November 28, 2012.

17 Institut für das Entgeltsystem im Krankenhaus. 2014. Definitionshandbuch 2015. www.g-drg.de/cms/G-DRG-System 2015/Definitionshandbuch/Definitionshandbuch_2015 Date last accessed: March 17, 2015. Date last updated: December 04, 2014.

18 Briggs AH, Weinstein MC, Fenwick EA, et al. Model parameter estimation and uncertainty analysis: a report of the ISPOR-SMDM Modeling Good Research Practices Task Force Working Group-6. Med Decis Making 2012; 32: 722-732.

19 Pym A, Diacon A, Conradie F, et al. Bedaquiline as part of a multi-drug resistant tuberculosis (MDR-TB) therapy regimen: final results of a single-arm, phase II trial (C209). OP-179-02. Int J Tuberc Lung Dis 2013; 17: Suppl. 2, S236.

20 Guglielmetti L, Le Dû D, Jachym M, et al. Compassionate use of bedaquiline for the treatment of multidrug-resistant and extensively drug-resistant tuberculosis: interim analysis of a French cohort. Clin Infect Dis 2015; 60: 188-194

21 Ndjeka N, Conradie F, Schnippel K, et al. Treatment of drug-resistant tuberculosis with bedaquiline in a high HIV prevalence setting: an interim cohort analysis. Int J Tuberc Lung Dis 2015; 19: 979-985.

22 Wolfson L, Wirth D, Jibbert J, et al. Costs and effectiveness of combination therapy with bedaquiline and other anti-tuberculosis drugs in patients with multi- and extensively drug-resistant tuberculosis in Germany. Value Health 2014; 17: A674-A675.

23 Destatis Statistisches Bundesamt. Aktuelle Sterbetafeln für das Frühere Bundesgebiet und die Neuen Länder. www.destatis.de/DE/ZahlenFakten/GesellschaftStaat/Bevoelkerung/Sterbefaelle/Tabellen/SterbetafelFBNL.html Date last accessed: March 17, 2015.

24 Liu CH, Li L, Chen Z, et al. Characteristics and treatment outcomes of patients with MDR and XDR tuberculosis in a TB referral hospital in Beijing: a 13-year experience. PLoS One 2011; 6: e19399.

25 Tiemersma EW, van der Werf MJ, Borgdorff MW, et al. Natural history of tuberculosis: duration and fatality of untreated pulmonary tuberculosis in HIV negative patients: a systematic review. PLoS One 2011; 6: e17601.

26 European Medicines Agency. Summary of opinion (initial authorisation): Deltyba. www.ema.europa.eu/docs/en GB/document_library/Summary_of_opinion_-_Initial_authorisation/human/002552/WC500155458.pdf Date last accessed: March 17, 2015. Date last updated: November 21, 2013.

\section{Comparison of the association with sleep apnoea of obesity versus insulin resistance}

To the Editor:

Obesity is considered to be both a risk factor for the development of obstructive sleep apnoea (OSA), and a contributor to the abnormal sleep patterns that characterise this syndrome [1]. Obesity is also associated with insulin resistance [2]. These two phenomena may be unrelated, and represent two untoward effects of 